\title{
Una pista polifónico-argumentativa para interpretar el agente en pasivas e impersonales con se
}

\author{
A polyphonic-argumentative clue to construe the agent in se passive and impersonal \\ Silvia Patricia Ramírez Gelbes \\ Universidad de San Andrés - Universidad de Buenos Aires - Buenos Aires - Argentina
}

\begin{abstract}
$\diamond$
Resumo: As frases deagentivadas são geralmente consideradas um recurso de discurso acadêmico para esconder o autor científica. No entanto, os agentes ausentes nem sempre correspondem com o palestrante. Em uma pesquisa, observou-se que os leitores deste tipo de discurso tendem a concordar sobre a interpretação do agente oculto: um agente particular que corresponde ao locutor, um agente particular que corresponde a alguém que não seja o locutor e um agente universal. Uma explicação da polifonia enunciativa é proposto aqui: os componentes da superfície discursiva em conjunto com as continuações plausíveis guiam para interpretar o agente oculto em passivos e impessoais, pelo menos neste gênero particular.
\end{abstract}

Palavras-chave: discurso acadêmico; polifonia; agente; passivo; impessoal

\begin{abstract}
Deagentived phrases are usually regarded as a device of academic discourse to hide the scientific author. However, missing agents do not always correspond with the speaker. In a survey, it was noted that readers of this type of discourse tend to agree on the interpretation of the hidden agent: a particular agent that corresponds to the speaker, a particular agent that corresponds to someone other than the speaker and a universal agent. An explanation from the enunciative polyphony is proposed here: the components of the discursive surface lead to plausible continuations and allow interpreting the hidden agent in passives and impersonals, at least in this particular genre.
\end{abstract}

Keywords: academic discourse; polyphony; agent; passive; impersonal

\section{Introducción}

Aunque la tradición bibliográfica suele sostener que el recurso a la sintaxis desagentivada en el discurso académico-científico (en adelante DAC) se debe estrictamente a la búsqueda de la objetividad, se defiende aquí la idea de que el borramiento del agente se relaciona, más vale, con los rasgos inherentes al ethos (AMOSSY, 1999; DUCROT, 1986; KERBRAT-ORECCHIONI, 2010; LE GUERN, 1978; MAINGUENEAU, 1996, 2002; entre muchos otros) propio del locutor académico. De hecho y dado que el DAC pretende, al mismo tiempo, comunicar un aporte científico y convencer a la comunidad del valor y la legitimidad de ese aporte, el locutor académico debe respetar dos condiciones básicas en la constitución del ethos del investigador, es decir, en la constitución de la imagen de sí que brinda por medio de su discurso: el decoro y la moderación (GARCÍA NEGRONI, 2006; RAMÍREZ GELBES, 2005a, 2006a,
2013), ${ }^{1}$ condiciones ambas que se manifiestan discursivamente por medio de la selección de opciones que, de modo consciente o no, hace el sujeto hablante. Y una de las opciones disponibles es, efectivamente, el ocultamiento del agente que lo representa (esto es, la primera persona), por medio de sintagmas desagentivados.

Sin embargo, los sintagmas desagentivados no solo opacan o silencian al agente locutor: también proveen otras posibilidades. A pesar de que algunos autores (por ejemplo Cinque [1988], para el caso de los predicados transitivos e intransitivos) afirman que la lectura del agente es arbitraria o inespecífica entre universal y particular en las construcciones desagentivadas, un trabajo previo (RAMÍREZ GELBES, 2006b) -que luego fue extendido a más informantes- mostró que los lectores tienden a

\footnotetext{
1 Como afirma Aristóteles en la Retórica, para persuadir, no alcanza con que el argumento sea convincente y fidedigno: es fundamental la actitud de quien produce el discurso y "que dé la impresión a los receptores de que se encuentra en determinada disposición respecto a ellos" (1998: 139).
} 
adjudicar una lectura definida al agente en cada uno de los casos particulares.

En efecto, a un grupo de sesenta informantes (21 lectores expertos de discurso académico y 39 estudiantes del último ciclo de un traductorado de inglés) se le administró una encuesta en la que se presentaban dos resúmenes de ponencias académicas con sintagmas desagentivados. Para cada uno de los casos de desagentivación destacados en ellas, se propusieron tres glosas posibles para interpretar al agente oculto: "todos", "el autor" y "alguien distinto del autor". Y se observó que la mayoría de los informantes seleccionaba la misma opción para cada caso particular. ${ }^{2}$ El hecho de que la mayoría de los informantes haya seleccionado la misma opción de paráfrasis permite inferir que efectivamente el propio discurso orienta a interpretar los agentes omitidos en cada caso de una manera definida, al menos en el género abstract de ponencia académica.

Distintas hipótesis han intentado dar cuenta del fenómeno (CHIERCHIA, 1995; CINQUE, 1988; MIGUEL, 1999; MENDIKOETXEA, 2002; MONTES GIRALDO, 2003; RAE 2009), aunque de modo poco específico. Es por ello que, en este trabajo, se ofrece una visión original que busca explicar esa lectura definida del agente desde la perspectiva de una teoría polifónica de la enunciación. Por medio de esta teoría, se mostrará que las distintas interpretaciones resultan asociadas con los puntos de vista puestos en escena por las diferentes ocurrencias de se desagentivado.

En lo que sigue, tras una breve revisión de las construcciones con se, se resume la encuesta que en 2004 se realizó con expertos y se amplió en 2007 con estudiantes de nivel superior, se presentan algunos estudios relativos a las pistas para la interpretación del agente en construcciones desagentivadas y se propone, finalmente, una respuesta al problema desde la perspectiva de la polifonía enunciativa.

\section{Algunas construcciones desagentivadas}

Como se sabe, las oraciones pasivas son aquellas cuyo sujeto gramatical es en realidad el objeto semántico o nocional (SÁNCHEZ LÓPEZ, 2002a), construidas con verbos diádicos o triádicos que dejan de requerir la manifestación del argumento externo por el tipo de construcción de la que se trata. Hay dos clases de oraciones pasivas: la pasiva perifrástica-que se construye con el verbo ser y el participio del verbo en cuestión concordado con el sujeto gramatical- y la pasiva con $s e^{3}$-que exige la presencia del clítico se y siempre aparece en $3^{\text {a }}$ persona-, también llamada pasiva refleja o pasiva impersonal (ver, por ejemplo, GILI y GAYA, 1948, p. 111 y ss.). Desde una perspectiva aspectual, Fernández Ramírez (1987) indica que, mientras la pasiva perifrástica se refiere generalmente a una acción singular, la pasiva refleja suele utilizarse para enunciar juicios de validez general. En cualquier caso, la pasiva perifrástica no se muestra compatible con ciertos verbos, ${ }^{4}$ en tanto la pasiva con se parece no tener restricciones de esa clase. Eso sí, mientras la pasiva perifrástica admite con comodidad la presencia del complemento agente, la pasiva refleja tiende fuertemente a rechazarlo. ${ }^{5}$

Tradicionalmente, de todos modos, se ha considerado que la característica más definida de las pasivas con se -en oposición a las perifrásticas- es el hecho de que su sujeto gramatical, por un lado, adopta una posición posverbal cuando no está marcado y, por el otro, tiene necesariamente que aparecer sin artículo determinante cuando se trata de un sintagma nominal (SN) que alude al rasgo "animado". Así se ve en (1).

(1) a. Se necesitan expertos.

b. \#Se necesitan los expertos. ${ }^{6}$

Las impersonales, por su parte, son las oraciones que no tienen sujeto gramatical, ya sea porque el predicado excluye el argumento externo -como en el caso de los verbos atmosféricos o del verbo haber-, ya sea porque el argumento externo no presenta una referencia definida y se trata de una ocurrencia en la que el argumento interno no existe (2a) o está representado por un complemento de persona $(2 b)$.

(2) a. En este país se trabaja bien.

b. Se recibe a las turistas con amabilidad.

Las impersonales con se se construyen con el clítico se y exigen una flexión verbal -desde una posición normativista- siempre en $3^{\text {a }}$ persona del singular. ${ }^{7}$ Las concordancias de los predicativos se realizan, por lo tanto, siempre en singular ${ }^{8} \mathrm{y}$, salvo indicación contextual pragmática (como es el caso de [3b]), en masculino:

(3) a. En este instituto se es protegido.

b. Cuando se está embarazada, se hinchan los pies.

\footnotetext{
2 El porcentaje restante, en general, se repartía de manera algo aleatoria, a excepcion del caso del ejemplo (14), que se estudia oportunamente.

3 El se de las construcciones desagentivadas - esto es, pasivas e impersonales- es llamado no paradigmático (SUÑER, 1973) porque no se relaciona paradigmáticamente con las formas reflexivas me, te y nos: las oraciones desagentivadas solo pueden ocurrir en tercera persona. Su uso ha originado controversias entre los investigadores y algunos, como el Esbozo, han sugerido que se trata de un pronombre con valor de sujeto indeterminado, similar al francés on o al alemán man, en tanto otros parecen sugerir que es simplemente un morfema verbal (MENDIKOETXEA, 1999, p. 1653).

4 Resultan raras algunas ocurrencias como "La canción fue cantada mal" o "Fue dicho que el Presidente no iría a la reunión". Más aún, el verbo tener, ineluctablemente diádico, no admite la ocurrencia en pasiva perifrástica en su acepción de posesión.

5 No es infrecuente en ella, con todo, la ocurrencia del complemento agente nucleado por la frase por parte de + grupo nominal, particularmente en el discurso judicial o burocrático (ver RAE, 2009, p. 3090).

6 Este ejemplo deja de ser agramatical si se le da una lectura recíproca, del tipo "Los expertos se necesitan mutuamente".

7 Para una discusión sobre este tema, ver Mendikoetxea (1999).

8 No ocurre lo mismo en italiano: "Non si é contenti".
} 
Para cada uno de los casos presentados, podría buscarse una glosa apropiada, a la manera de los ejemplos siguientes:

(1) Se necesitan expertos.

Es probablemente interpretado como alguien necesita expertos.

(2) En este país se trabaja bien.

Es probablemente interpretado como en este país todos trabajamos bien.

(3) Se recibe a las turistas con amabilidad.

Es probablemente interpretado como todos recibimos a las turistas con amabilidad.

(4) En este instituto se es protegido.

Es probablemente interpretado como en este instituto, alguien protege (a los demás).

(5) Cuando se está embarazada, se hinchan los pies

Es probablemente interpretado como cuando cualquier mujer está embarazada, se le hinchan los pies.

Y estas glosas, en coincidencia con las propuestas disponibles, no discriminan más que entre lecturas genéricas y particulares. Sin embargo, en contexto, los lectores expertos y semiexpertos en lectura de DAC son capaces de interpretar el agente de manera definida en resúmenes de ponencias entre una opción genérica ("todos hacemos esto") y dos opciones particulares ("el autor del texto hace esto" y "alguien distinto del autor del texto hace esto"). ${ }^{9}$ Ello autoriza a conjeturar que los agentes de estas construcciones pueden ser repuestos a partir de ciertas pistas provistas por el propio texto.

En lo que sigue, presentaré los resultados de una encuesta de lectura del agente que se llevó adelante en dos etapas: con lectores expertos (científicos) en 2004 y con lectores semiexpertos (estudiantes del último tramo de nivel superior) en 2007.

\footnotetext{
9 Existe una cuarta posibilidad, la que se corresponde con un se que puede interpretarse como alusión al lector, tal cual ocurre en el caso prototípico de Véase el siguiente ejemplo o de Considérense estos casos. En estas ocurrencias, aunque se trata estrictamente de pasivas con se tal cual muestra la concordancia de número con el sujeto (i.e., el objeto nocional, como se explicó más arriba), el subjuntivo orienta a la interpretación en su valor de imperativo (y el imperativo, se sabe, solo puede concebirse en términos de la segunda persona). Si no resulta examinada aquí es porque esa opción no fue hallada en el corpus de resúmenes (y hasta puede presumirse que sea infrecuente en ese género). El tema será tratado en un trabajo que contemple otros géneros académicos.

${ }^{10}$ Estos 2 abstracts fueron seleccionados entre 300, por resultar ejemplares para la muestra, dado que remitían a los tres distintos tipos de agentes hallados en ese corpus y descriptos más arriba.

11 El ejercicio se registra en el apéndice.

12 Por ejemplo, dado "Se sabe esto", las opciones eran "Todos sabemos esto", "El autor sabe esto", "Alguien distinto del autor sabe esto".
}

\section{La lectura del agente}

En 2004 y en 2007, administré a un total de 60 informantes una encuesta relativa a la interpretación del agente en sintagmas con se pasivo o impersonal. Luego de una prueba preliminar llevada con cinco voluntarios, se seleccionaron 2 abstracts académicos de lingüística, ${ }^{10}$ tomados del volumen Resúmenes de comunicaciones del "Segundo Coloquio Latinoamericano de Analistas del Discurso" (La Plata, 1998), para elaborar el ejercicio de reconocimiento. ${ }^{11}$ Los informantes debían seleccionar la opción de reposición del agente que les pareciera acertada. Los ítems encuestados se distribuyeron de la siguiente manera: cuatro en el primer resumen y cuatro en el segundo, lo que constituye un total de ocho preguntas.

Dicho en otros términos, esa tarea era un ejercicio de respuesta múltiple en el que, dado "Se $p$ ", se ofrecían tres opciones, del siguiente tipo:
a. "Todos hacemos $p$ "
b. "El autor del texto hace $p$ "
c. "Alguien distinto del autor del texto hace $p$ "12

o, como los nombro más técnicamente,

a. una lectura de agente particular o individual que se corresponde con el locutor: se locutivo

b. una lectura de agente particular o individual que se corresponde con alguien distinto del locutor: se alterlocutivo

c. una lectura de agente universal o general: se universal.

Veamos los casos concretos en uno de los abstracts con encuesta presentados a los informantes:

\section{Revisión del concepto de "evaluación" narrativa: una perspectiva global}

La evaluación parece ser la sección de la narración de la cual depende el interés de una historia. En el trabajo de Labov (1972) sobre la narración oral, se establece un continuo con diferentes tipos de evaluación que van desde las más específicas hasta las más elaboradas, que el autor denomina evaluación externa, subordinación de la evaluación, acción evaluativa y suspensión de la acción.

En esta oportunidad, se propone un ordenamiento distinto de los diferentes tipos de evaluación. Se deja de lado su grado de elaboración para pasar a considerar la relación de las diferentes categorías evaluativas, tanto con las funciones del lenguaje como con las teorías sobre el punto de vista. De esta manera se logra una visión más coherente de esta sección de la narración.

1.a. ... se establece un continuo con diferentes tipos de evaluación...

a. Todos establecemos un continuo referido a la evaluación narrativa con diferentes tipos de evaluación. 
b. El autor de este texto establece un continuo referido a la evaluación narrativa con diferentes tipos de evaluación.

c. Alguien distinto del autor de este texto establece un continuo referido a la evaluación narrativa con diferentes tipos de evaluación. (se alterlocutivo) ${ }^{13}$

1.b.... se propone un ordenamiento distinto..

a. Todos proponemos un ordenamiento distinto de los diferentes tipos de evaluación.

b. El autor de este texto propone un ordenamiento distinto de los diferentes tipos de evaluación. (se locutivo)

c. Alguien distinto del autor de este texto propone un ordenamiento distinto de los diferentes tipos de evaluación.

1.c. Se deja de lado su grado de elaboración...

a. Todos dejamos de lado el grado de elaboración de los distintos tipos de evaluación narrativa para pasar a considerar la relación de las diferentes categorías evaluativas.

b. El autor de este texto deja de lado el grado de elaboración de los distintos tipos de evaluación narrativa para pasar a considerar la relación de las diferentes categorías evaluativas. (se locutivo)

c. Alguien distinto del autor de este texto deja de lado el grado de elaboración de los distintos tipos de evaluación narrativa para pasar a considerar la relación de las diferentes categorías evaluativas.

1.d....se logra una visión más coherente...

a. De esta manera, todos logramos una visión más coherente de esta sección de la narración. (se universal)

b. De esta manera, el autor de este texto logra una visión más coherente de esta sección de la narración.

c. De esta manera, alguien distinto del autor de este texto logra una visión más coherente de esta sección de la narración.

Como resultado, pudo observarse que la coincidencia en la selección de una de las opciones para cada ejemplo era significativamente alta en la mayoría de los casos:
En efecto y según se ve, de los ocho casos presentados, hubo un ciento por ciento de coincidencia en la interpretación del agente en uno de los casos (el 1.c.), un noventa o un noventa y cinco por ciento de coincidencia en cuatro casos (el 1.b., el 2.a., el 2.b. y el 2.c.), un setenta y nueve por ciento de coincidencia en un caso (el 1.a.) y sesenta y ocho por ciento (el 2.d.) y cincuenta y cuatro por ciento (el 1.d.) en sendos casos.

Glosado, entonces, el ítem 1.a. que presento para 1. ("Revisión del concepto de "evaluación" narrativa: una perspectiva global"), la lectura que se obtuvo fue la siguiente:

La evaluación parece ser la sección de la narración de la cual depende el interés de una historia. En el trabajo de Labov (1972) sobre la narración oral, se establece un continuo con diferentes tipos de evaluación que van desde las más específicas hasta las más elaboradas, que el autor denomina evaluación externa, subordinación de la evaluación, acción evaluativa y suspensión de la acción.

fue interpretado por 47 informantes como:

La evaluación parece ser la sección de la narración de la cual depende el interés de una historia. En el trabajo de Labov (1972) sobre la narración oral, alguien distinto del autor de este texto establece un continuo con diferentes tipos de evaluación que van desde las más específicas hasta las más elaboradas, que el autor denomina evaluación externa, subordinación de la evaluación, acción evaluativa y suspensión de la acción.

En el parágrafo que sigue, se registrarán las hipótesis propuestas por algunos autores acerca de cuáles son las razones que inducen a la interpretación de los agentes silenciados en pasivas e impersonales con se.

\section{Las pistas que permiten recuperar el agente}

Tanto pasivas como impersonales carecen de sujeto semántico o nocional explícito, ${ }^{14}$ si bien este puede explicitarse por medio de un adjunto introducido

\begin{tabular}{|c|c|c|c|c|c|c|c|c|}
\hline Item & 1.a. & 1.b. & 1.c. & 1.d. & 2.a. & 2.b. & 2.c. & 2.d. \\
\hline \multirow{2}{*}{$\begin{array}{l}\text { Porcentaje de coincidencia } \\
\text { en la lectura }\end{array}$} & 79 & 90 & 100 & 54 & 90 & 90 & 95 & 68 \\
\hline & 47 inform & 54 inform & 60 inform & 32 inform & 54 inform & 54 inform & 57 inform & 41 inform \\
\hline
\end{tabular}

\footnotetext{
13 Señalo en cursiva negrita y con su nombre técnico la opción preferida por mayor cantidad de lectores. Hago lo mismo en el apéndice.

14 Con la expresión "sujeto nocional o semántico" se evitan las restricciones que impone el uso del término "agente" frente a otras posibilidades efectivas como puede ser, por ejemplo, el experimentante. Aun así, se emplea aquí el término "agente" del modo amplio en que se usa, en
}

\footnotetext{
general, la expresión "complemento agente", o sea, englobando en é los papeles temáticos que ocupan el espacio del argumento externo. Por otra parte, la disquisición acerca de las propiedades argumentales del agente - esto es, si se trata de un argumento o de un adjunto- no es tratada aquí, dado que excede los alcances de este trabajo (para una discusión al respecto, ver, por ejemplo, RAE, 2009, p. 3050 y ss.)
} 
por por. ${ }^{15}$ En los casos en que no aparece explicitado, sin embargo, la bibliografía (entre otros, CINQUE, 1988; CHIERCHIA, 1995; MIGUEL, 1999; MENDIKOETXEA, 2002; MONTES GIRALDO, 2003; RAE, 2009) propone que el argumento agente implicado tiene una lectura ya sea universal o general, ya sea particular o individual, pero inespecífica. ${ }^{16}$

Según esas hipótesis, la lectura universal aparece relacionada con una interpretación compatible con formas como "todo el mundo", "uno" o "cualquiera", mientras que la lectura particular se corresponde con una forma individual que podría estar representada por "alguien", "alguno" o "cierta gente".

Ahora bien, ambas interpretaciones no son realmente aleatorias ni simplemente arbitrarias. De hecho, distintos autores adjudican esa interpretación universal o particular a distintas condiciones: la naturaleza temporal-aspectual del predicado, las características argumentales del predicado, las restricciones que imponen los elementos presentes en la frase.

La primera hipótesis es la que sostiene Miguel (1992, 1999). Ella afirma que los verbos no agentivos (sean estos estativos o dinámicos) en construcción con se solo admiten una lectura universal. Los verbos agentivos con se pasivo o impersonal, en cambio, son interpretados como de agente general cuando "el evento denotado por el predicado es válido para cualquier momento del tiempo -lectura favorecida por la forma verbal no perfecta del tiempo-" (MIGUEL, 1999, p. 3051). Por su parte, cuando "la referencia temporal-aspectual ${ }^{17}$ de la oración [con se] alude a un momento específico, puntual y acabado" (p. 3051), la construcción es interpretada como de agente particular.

En línea con esta postura, la RAE (2009) indica que hay dos clases de interpretaciones: las genéricas, que se construyen en presente, imperfecto y otros tiempos imperfectivos, del tipo universal; y las existenciales o episódicas, que admiten tiempos perfectivos y por las que "el agente suele estar presente en la mente del que habla" (RAE, 2009, p. 3084). Asimismo, se afirma allí que las impersonales con se pueden incluir o excluir al hablante; cuando lo incluyen, resultan similares a las oraciones construidas con el pronombre uno. $^{18}$

\footnotetext{
15 Ver nota 3.

16 Otro trabajo (RAMÍREZ GELBES, 2005b) estudia la recuperación del agente/experimentante/ paciente en títulos de papers, i.e., no ya en los sintagmas desagentivados representados por pasivas o impersonales con $s e$, sino en sintagmas nucleados por una nominalización. Se observa allí que la lectura descrimina claramente entre lectores expertos y semiexpertos por un lado y legos por el otro. Esto sugiere que la interpretación del agente resulta bastante condicionada por la experticia en la lectura.

17 Al hablar de naturaleza o condición temporal-aspectual (o tempoaspectual), Miguel (1999) se refiere al aspecto que se manifiesta como integrante de la flexión verbal. Esta misma descripción se adopta aquí, para oponerla a la naturaleza o condición aspectual, que alude a las características propias de la aspectualidad o aspecto léxico o Aktionsart.
}

Cinque (1988) sostiene que la diferencia en la lectura se debe a que la naturaleza no argumental de los verbos inacusativos y estativos les impide tener una lectura de agente particular, en tanto que la naturaleza argumental de transitivos e intransitivos admite la doble lectura: universal o particular.

Chierchia (1995) observa que los adjuntos tienden a ser definitorios en la lectura del agente. En su análisis de la interpretación de PRO (el agente) en las construcciones desagentivadas, observa que, sin especificación provista por un adjunto, hay tendencia a obtener una lectura universal que incluye al autor del texto o locutor, en tanto que la presencia de adjuntos - como, por ejemplo, las cláusulas condicionales- tiende a orientar la lectura hacia el particular. Ahora bien, cuando la variable que incide es la de tiempo, el presente promueve la lectura universal, en tanto el pretérito perfecto promueve la lectura particular (en lo que termina coincidiendo con Miguel [1999] y con la RAE [2009]). Finalmente, Mendikoetxea (2002, p. 268) apunta que "la interpretación existencial de PRO surge cuando la construcción con se se predica de un argumento locativo Loc, que es parte de la estructura argumental de los predicados episódicos".

Frente a estos análisis, existen algunas indicaciones -si bien son pocas- acerca de que la lectura del agente en las oraciones pasivas e impersonales con se se realiza de manera más definida. Así, Montes Giraldo (2003, p. 124) -sin ofrecer ningún tipo de justificación-clasifica los usos de se y reconoce la existencia de un "impersonal en que el se elude la mención del agente, pero este es fácilmente precisable" -algo similar al "agente que está en la mente" de la RAE (2009)-, en oposición a un caso de desagentivación por la que el agente se diluye en una vaga generalidad.

Como se ve, estos razonamientos no explican la causa de las diferentes interpretaciones ni contemplan una discriminación entre distintas opciones particulares. No obstante ellos, los resultados de esa encuesta, restringida a un ámbito genérico y llevada a cabo entre lectores expertos y semiexpertos de DAC, parecen mostrar que pueden existir lecturas más definidas cuando se trata de interpretar el agente oculto en algunas construcciones

\footnotetext{
18 Además de las pasivas e impersonales con se, otros dos tipos de construcciones con verbo conjugado ocultan el agente: las impersonales con sujeto arbitrario de segunda persona (como en Cuando vas a hacer un trámite y te tratan mal, te enojás y protestás) y las impersonales con sujeto tácito de interpretación inespecífica (como en Dicen que aumentará el dólar). Acerca de los sujetos arbitrarios de segunda persona (sujetos que, aunque aparecen en segunda persona singular y pueden estar explícitos, se caracterizan por carecer de contenido referencial), Hernanz (1990, p. 160) afirma que los agentes "adoptan valores próximos en algunos casos a la cuantificación universal, mientras que en otros son prácticamente identificables con un 'yo' encubierto". En cuanto a los casos de sujeto tácito con interpretación inespecífica, el verbo siempre aparece en tercera persona del plural y en voz activa, el referente del sujeto tácito es necesariamente humano y la interpretación del agente excluye ineludiblemente al locutor (ver RAE, 2009, p. 3077 y ss.)
} 
desagentivadas. En lo que sigue, me ocuparé de las pistas para obtener esas lecturas, desde una óptica ostensiblemente distinta.

\section{La Teoría de la Polifonía ${ }^{19}$ y la lectura del agente}

Tal cual queda dicho, las explicaciones que se han brindado hasta el momento no logran dar respuesta a las diferentes lecturas del agente que los interpretantes hacen de los enunciados impersonales reflejos (pasivas e impersonales con se). Frente a ellas, la perspectiva de la polifonía enunciativa, teoría no referencialista que recusa las concepciones veritativistas de la semántica, ofrece un marco propicio para comprender el fenómeno.

Entendida desde el estudio del discurso, la polifonía rechaza la noción de unicidad del sujeto discursivo y postula, para caracterizarlo, la imagen de una escena en la que emergen diferentes puntos de vista. Como sostiene Anscombre (2008, p. 30), tres son los niveles que distingue toda teoría polifónica: el nivel de la producción del enunciado, el nivel de la responsabilidad del enunciado y el nivel de los puntos de vista que pone en escena el enunciado.

El primer nivel corresponde al sujeto hablante, el individuo de carne y hueso que efectúa el acto de proferir el enunciado. Este sujeto no interesará a la hora de llevar adelante este análisis. El segundo nivel se corresponde con el locutor. El locutor es responsable del enunciado y es además responsable de la escena en que emergen los diversos puntos de vista. Pues bien, debe notarse que el locutor es responsable del enunciado dentro del propio discurso, y eso significa que no ha de confundirse esta noción con la de responsable legal del enunciado, o sea, con la del sujeto hablante. Si el sujeto hablante tiene que hacerse cargo de sus palabras ante la justicia, por ejemplo, no hablaremos allí de locutor, más allá de que se considere al locutor del enunciado de marras para juzgar al sujeto de carne y hueso.

En los términos de Ducrot (1986) -que serán los que seguiremos aquí-, el locutor se constituye por medio de dos instancias: el L o locutor responsable de la enunciación (locutor en tanto tal) y el $\lambda$ o locutor como ser en el mundo. El enunciado manifiesta marcas que evidencian al locutor, y estas marcas se vinculan tanto con la deixis personal cuanto con la deixis espacial y temporal, siempre referidas a la primera persona: yo/mel mími/conmigo/este/aqui/ahora.

\footnotetext{
${ }^{19}$ Como afirma Anscombre (2008), no debería hablarse de la teoría de la polifonía sino de las teorías de la polifonía, desde el momento en que son varias las teorías lingüístico-discursivas que se inspiran en la propuesta de Bajtín (1986), quien aplicó el concepto a la literatura.
}

El tercer nivel, finalmente, es el nivel de los enunciadores o puntos de vista. Los enunciadores son, en efecto, las imágenes responsables de los diversos puntos de vista evocados en el enunciado. Pero es L quien resulta responsable de la escena general. Eso sí, no es necesario que esté de acuerdo con los distintos puntos de vista que se ponen en escena: puede de hecho identificarse con alguno, pero puede también rechazarlo, distanciarse, rectificarlo, descalificarlo o solo acordar con él. Desde esta perspectiva, propongo que los puntos de vista de los enunciadores serán percibidos por el lector del DAC como instrucciones para interpretar el agente en los sintagmas desagentivados.

Para constatar los distintos puntos de vista puestos en escena y el posicionamiento que L adopta en relación con ellos, se brindará aquí una especie de fórmula argumentativa: cada segmento efectivo será continuado a la derecha en bastardilla con una paráfrasis en primera persona. Cuando quiera demostrarse la identificación, se relacionarán ambos segmentos por medio de un marcador de reformulación parafrástica (GARCÍA NEGRONI 2009) como en otras palabras. Cuando quiera demostrarse el acuerdo, se empleará un consecutivo como así que. Cuando quiera demostrarse la atenuación, se empleará un marcador de atenuación como al menos. Cuando quiera demostrarse el distanciamiento, el desacuerdo o el rechazo, se empleará un conector adversativo como pero y se explicitará en primera persona el distanciamiento, desacuerdo o rechazo.

Así, con estas fórmulas, se analizarán a continuación los casos 2.a., 1.a. y 1.d. correspondientes a los resúmenes con los que se trabajó en la encuesta (ver Apéndice).

Caso 2.a. lectura de agente particular o individual que se corresponde con el locutor: se locutivo

(9) Este trabajo se propone alcanzar una definición del concepto de "texto" dentro del campo especulativo de la Teoría Musical. Para lograrlo, se considerará la organización y estructura del texto musical desde un enfoque interdisciplinario, incorporando la perspectiva de la Lingüística textual. Se analizarán posibles paralelismos entre el texto del lenguaje natural y el texto del lenguaje musical, a fin de verificar si las categorías y los procedimientos de análisis que definen al primero pueden ser transferidos al segundo.

Desde luego, la presencia de "este trabajo" en el cotexto previo orienta - por el propio peso deíctico del "aquí y ahora" propios de la primera persona- hacia una lectura de identificación entre el punto de vista de ese se y L, como muestra (9a). Concomitantemente, la imposibilidad de distanciamiento o, más aún, de desacuerdo (9b) o incluso de rechazo (9c) de ese punto de 
vista por parte de $\mathrm{L}$, fortalece -o evidencia- la lectura de se locutivo, en la medida en que obliga a solapar el punto de vista que emerge en ese se con la del locutor-autor, todo ello expuesto en (9a) a (9c).

(9) Este trabajo se propone... Para lograrlo, se considerará la organización y estructura del texto musical desde un enfoque interdisciplinario.

a. Este trabajo se propone... Para lograrlo, se considerará la organización y estructura del texto musical desde un enfoque interdisciplinario. En otras palabras, tomaré en consideración la macroestructura del texto musical desde una perspectiva interdisciplinaria.

b. Este trabajo se propone... Para lograrlo, se considerará la organización y estructura del texto musical desde un enfoque interdisciplinario, \#pero no estoy de acuerdo con tomar en consideración la organización y estructura del texto musical desde una perspectiva interdisciplinaria.

c. Este trabajo se propone... Para lograrlo, se considerará la organización y estructura del texto musical desde un enfoque interdisciplinario, \#pero no tomaré en consideración la organización y estructura del texto musical desde una perspectiva interdisciplinaria.

Obsérvese, eso sí, que (9b) y (9c) pueden efectivamente ocurrir (y por eso el signo numeral) toda vez que "este trabajo" adopte un sentido anafórico y no deíctico, i.e., el sentido de una referencia cohesiva al último trabajo mencionado en el cotexto previo, del que L podría distanciarse: de ser así, no hablaríamos de identificación de $\mathrm{L}$ con el punto de vista de ese se, sino de alguna forma de distanciamiento, similar a lo que se verá más abajo en (12). No es este, evidentemente, el sentido del enunciado presente en 2.a.

Esta explicación puede hacerse extensiva a los otros casos leídos como se locutivo en la encuesta que sirve de base a este trabajo y que se ofrecen aquí como (10) y (11).

(10) En esta oportunidad, se propone un ordenamiento distinto de los diferentes tipos de evaluación.

a. En esta oportunidad, se propone un ordenamiento distinto de los diferentes tipos de evaluación. En otras palabras, propongo ordenar de otro modo las distintas clases de evaluación.

b. En esta oportunidad, se propone un ordenamiento distinto de los diferentes tipos de evaluación, \#pero no estoy de acuerdo con ordenar de otro modo las distintas clases de evaluación.

c. En esta oportunidad, se propone un ordenamiento distinto de los diferentes tipos de evaluación, \#pero no propongo ordenar de otro modo las distintas clases de evaluación.
(11) En esta oportunidad... Se deja de lado su grado de elaboración para pasar a considerar la relación de las diferentes categorías evaluativas.

a. En esta oportunidad... Se deja de lado su grado de elaboración para pasar a considerar la relación de las diferentes categorías evaluativas. En otras palabras, me aparto de su grado de elaboración y me ocupo de las diferentes categorías evaluativas.

b. En esta oportunidad... Se deja de lado su grado de elaboración para pasar a considerar la relación de las diferentes categorías evaluativas, \#pero no estoy de acuerdo con dejar de lado su grado de elaboración para ocuparme de las diferentes categorias evaluativas.

c. En esta oportunidad... Se deja de lado su grado de elaboración para pasar a considerar la relación de las diferentes categorías evaluativas, \#pero no dejo de lado su grado de elaboración para ocuparme de las diferentes categorías evaluativas.

Como se ve en las continuaciones a la derecha presentadas en (10a) y (11a), el L representado por la primera persona de los verbos y por me obliga a interpretar la identificación entre el se del segmento a la izquierda y $\mathrm{L}$, mientras que la incoherencia evidenciada en las continuaciones de (b) y (c) exige admitir que L no puede ni distanciarse ni rechazar el punto de vista del se correspondiente.

Caso 1.a. lectura de agente particular o individual que se corresponde con alguien distinto del locutor: se alterlocutivo

(12) La evaluación parece ser la sección de la narración de la cual depende el interés de una historia. En el trabajo de Labov (1972) sobre la narración oral, se establece un continuo con diferentes tipos de evaluación que van desde las más específicas hasta las más elaboradas, que el autor denomina evaluación externa, subordinación de la evaluación, acción evaluativa y suspensión de la acción.

En esta oportunidad, se propone un ordenamiento distinto de los diferentes tipos de evaluación. Se deja de lado su grado de elaboración para pasar a considerar la relación de las diferentes categorías evaluativas, tanto con las funciones del lenguaje como con las teorías sobre el punto de vista. De esta manera se logra una visión más coherente de esta sección de la narración.

En este caso, dos son las marcas de la superficie discursiva que orientan inicialmente a reconocer la presencia de un punto de vista con el que $\mathrm{L}$ no se identifica. En primer lugar, la mención directa de la fuente de ese discurso, i.e., Labov (1972). En segundo lugar, el 
contraste deíctico que obliga a reconocer la distancia con "ese trabajo" a partir de la continuación tempolocativa expresada por "esta oportunidad".

Pero no solo eso. A diferencia del caso anterior (9), algunas continuaciones plausibles nos permiten advertir que L puede tanto acordar con ese punto de vista (12a) cuanto tomar distancia del punto de vista puesto en escena ([12b] y [12c]) cuando se habla del trabajo de Labov, pero lo que no puede hacer es identificarse con él, como muestra la imposibilidad de la reformulación parafrástica con la primera persona del singular y en otras palabras (12d).

(12) En el trabajo de Labov (1972) sobre la narración oral, se establece un continuo con diferentes tipos de evaluación que van desde las más específicas hasta las más elaboradas... En esta oportunidad...

a. En el trabajo de Labov (1972) sobre la narración oral, se establece un continuo con diferentes tipos de evaluación que van desde las más específicas hasta las más elaboradas, $y$ estoy de acuerdo con la propuesta de Labov.

b. En el trabajo de Labov (1972) sobre la narración oral, se establece un continuo con diferentes tipos de evaluación que van desde las más específicas hasta las más elaboradas, pero no estoy de acuerdo con la propuesta de Labov.

c. En el trabajo de Labov (1972) sobre la narración oral, se establece un continuo con diferentes tipos de evaluación que van desde las más específicas hasta las más elaboradas, pero yo no establezco ningún continuo.

d. En el trabajo de Labov (1972) sobre la narración oral, se establece un continuo con diferentes tipos de evaluación que van desde las más específicas hasta las más elaboradas. *En otras palabras, elaboro una gradación con tipos de evaluación que van desde las más especificas a las más elaboradas.

Si bien no se presentaron otros casos de alterlocutivo en nuestra encuesta inicial, lo cierto es que la existencia de estos ejemplos no es infrecuente en el discurso académico, que debe confesar siempre -a riesgo de ser calificado de plagiario si no lo hace- el origen de los discursos ajenos al locutor-autor.

(13) Se pueden reconocer dos niveles de adverbios (Cinque 1997).

a. Se pueden reconocer dos niveles de adverbios (Cinque 1997), y estoy de acuerdo con esa propuesta.

b. Se pueden reconocer dos niveles de adverbios (Cinque 1997), pero no estoy de acuerdo con esa propuesta. c. Se pueden reconocer dos niveles de adverbios (Cinque 1997), pero no los reconozco aquí.

d. Se pueden reconocer dos niveles de adverbios (Cinque 1997). \#En otras palabras, distingo dos clases adverbiales en distintos niveles.

Nótese que, a pesar de que los casos (13a) a (13c) resultan claros, es necesario explicar mejor lo que ocurre con (13d). La continuación propuesta en (13d) no resulta incoherente si el se se lee como locutivo y la indicación entre paréntesis señala el acuerdo con el autor que ha formulado la afirmación ("hay dos niveles de adverbios") con anterioridad. Ante la ausencia de continuaciones, sin embargo, habrá tendencia a interpretar ese se simplemente como alterlocutivo.

Por otra parte, cuando el discurso atribuible a otra(s) fuente(s) no ofrece indicación de origen, el agente será probablemente interpretado como un universal, según se muestra en los ejemplos (14) y (15).

\section{Caso 1.d. lectura de agente universal o general: se universal}

(14) En esta oportunidad, se propone un ordenamiento distinto de los diferentes tipos de evaluación. Se deja de lado su grado de elaboración para pasar a considerar la relación de las diferentes categorías evaluativas, tanto con las funciones del lenguaje como con las teorías sobre el punto de vista. De esta ${ }^{20}$ manera, se logra una visión más coherente de esta ${ }^{21}$ sección de la narración.

Los casos de se que carecen de indicaciones de fuente del discurso y de instrucciones deícticas que permitan situarlo en relación con el "aquí y ahora" de L, como (14), orientan a leer el se con el sentido que más se ha considerado y estudiado en la perspectiva de la polifonía: el se como la voz de la doxa. Estos enunciados -asociados a la historia por Benveniste (1966)- no tienen locutor e incluyen al $\lambda$ como incorporado en la comunidad indefinida que esa voz encarna.

La inexistencia de otras indicaciones discursivas, en efecto, obliga a leer este se como proveniente de una voz difusa, no localizada, que incluso ha sido rotulada como la voz del Omni-locutor (ANSCOMBRE, 1995, 2008) o de la sabiduría popular. Cabe destacar que este se admite diversos posicionamientos de $\mathrm{L}$ en relación con ese enunciador o punto de vista denominado Omnilocutor: 22

\footnotetext{
${ }^{20}$ Es importante destacar aquí que este demostrativo no resulta deíctico sino anafórico, por lo que no debe tomarse como marca de localización respecto del L.

21 Ídem nota anterior.

22 Excepción hecha de la identificación, que lo transformaría en un se locutivo. Ver, al respecto, (16).
} 
(14)

a. De esta manera, se logra una visión más coherente de esta sección de la narración, así que reconozco esa coherencia. [acuerdo]

b. De esta manera, se logra una visión más coherente de esta sección de la narración, pero no puedo reconocerla en este trabajo. [distanciamiento]

c. De esta manera, se logra una visión más coherente de esta sección de la narración, al menos, eso creo. [atenuación]

d. De esta manera, se logra una visión más coherente de esta sección de la narración, *pero yo no lo logro. [rechazo]

Ahora bien, si es cierto que estos posicionamientos son todos admisibles cuando el discurso pone en escena la voz de la doxa, también es cierto que la lectura del se es aceptable solo si no resulta desafiada. Obsérvese que, si bien en (14b) el logro de una visión más coherente se presenta como impracticable en esta ocasión y en (14c) resulta morigerado, la aseveración "de esta manera, se logra una visión más coherente de esta sección" incluye en su generalidad a $\lambda$ y refleja el acuerdo -aun parcial- de L. Esa inclusión y ese reflejo se encuentran patentizados en la imposibilidad de (14d), que implicaría a un tiempo que el se no incluye a $\lambda$ y que $L$ rechaza ese punto de vista. Y es que, si la voz del se resultara desafiada en la continuación, como ocurre en (15),

(15) Suele afirmarse que el inglés es una lengua de cultura, pero yo no lo afirmo.

no se hablaría de un se universal sino de un alterlocutivo. Y, en rigor de verdad, correspondería -según las normas de ética del DAC-indicar entre paréntesis quiénes son los autores que afirman que el inglés es una lengua de cultura.

Pero hay algo más aún. El caso (14) es el que obtuvo la menor coincidencia de lectura del agente: solo un 54\% de informantes (32 individuos) lo interpretaron como un universal. Frente a ellos, 26 informantes (el 44\% del total $^{23}$ leyeron este se como locutivo, es decir, el que se corresponde con la glosa "el autor de este texto logra una visión más coherente", como la interpretación que se ofrece en (16).

(16) De esta manera, se logra una visión más coherente de esta sección de la narración. En otras palabras, consigo una visión más consistente de este segmento. [identificación]

Según la perspectiva que se viene propiciando, este resultado parece razonable. Por un lado, el $54 \%$ de los informantes interpretó que ese $s e$, sin mayores indicaciones

\footnotetext{
${ }^{23}$ Dos informantes no respondieron.
}

deícticas en el cotexto inmediato ni continuaciones opositivas, se corresponde con el universal inclusivo. El $44 \%$ de los informantes, por el contrario, mantuvo activo el sintagma "en esta oportunidad", que, aunque no se hallaba en el cotexto inmediato, abría el párrafo del se de marras. No ha de olvidarse, además, que la ocurrencia de se inmediatamente previa a esta, nombrada como 1.c. y (11), recibió un $100 \%$ de coincidencia acerca de su interpretación como locutivo, otro dato más para habilitar esa misma lectura en 1.d. (14).

Por todo lo visto hasta aquí, un resumen tentativo del tema en el marco de esta teoría polifónica podría ser el siguiente:

a) SE LOCUTIVO: L se identifica con el punto de vista puesto en escena por el se; hay indicaciones deícticas de primera persona que acreditan esa identificación.

b) SE ALTERLOCUTIVO: L acuerda, se distancia, discute, rechaza o refuta el punto de vista puesto en escena por el se; hay indicación del origen o fuente del discurso (siempre ajeno al autor).

c) SE UNIVERSAL: L acuerda con el punto de vista de ese se que pone en escena una voz indefinida; no hay marcaciones deícticas ni de fuente del discurso.

En definitiva, y aunque las pistas para la recuperación del agente no estén exentas de ambigüedades, como ocurre con el caso considerado en (14), los componentes de la superficie discursiva y las continuaciones argumentativas plausibles de los segmentos actúan solidariamente a la hora de evidenciar los puntos de vista que quedan expresados en la escena del discurso. Y, dado que tanto los lectores expertos cuanto los estudiantes de nivel superior se hallan familiarizados con estos indicadores gracias a la alta exposición que han tenido a este tipo de discurso es que, en términos generales -aunque no absolutos- puede observarse una notable coincidencia en la recuperación de los agentes que las ocurrencias desagentivadas de se ocultan.

\section{Conclusiones}

En una encuesta administrada a 60 informantes expertos o semiexpertos en la lectura de discurso académico, se reveló una fuerte tendencia a coincidir en la interpretación del agente correspondiente a construcciones desagentivadas (pasivas e impersonales con se) entre tres opciones disponibles: el autor, alguien distinto del autor, todos.

Este resultado, distanciado de las explicaciones habituales que concentran en dos las opciones, fue analizado en este trabajo a la luz de una teoría polifónica enunciativa. En esa línea, se observó que los componentes de la superficie discursiva (por ejemplo, las marcas 
deícticas de primera persona o las indicaciones de la fuente del discurso) juntamente con las continuaciones argumentativas plausibles de los segmentos (por caso, en términos de acuerdo u oposición) orientan a los lectores a interpretar una $u$ otra posibilidad.

Es necesario, sin embargo, hacer una salvedad. Aunque la tendencia explicitada por la coincidencia en las lecturas autoriza a hablar de interpretaciones definidas del agente en las construcciones desagentivadas con se, debe reconocerse la existencia de casos en los cuales existe una cuota de ambigüedad manifiesta que no permite definir con claridad, incluso cuando hablemos de lectores expertos, de qué se se trata. Aun así, las pruebas aportadas aquí permiten, como mínimo, revisar las hipótesis previas.

En definitiva, los sintagmas desagentivados no son un medio del DAC para manifestar la búsqueda de la objetividad sino, más vale, el recurso discursivo, específico de este tipo de retórica, que permite poner en juego los distintos puntos de vista que interactúan en el texto cuando esos puntos de vista no adoptan el papel evidenciador de los sujetos sintácticos. Y los lectores emparentados con su lectura resultan competentes en la interpretación de esa escena discursiva.

\section{Referencias}

AMOSSY, Ruth (ed.). Images de soi dans le discours. La construction de l'ethos. Lausanne: Delachaux et Niestlé, 1999.

ANSCOMBRE, Jean-Claude. La polifonía: nociones y problemas. AO, LVIII, tomo 58-59, p. 21-51, 2008-2009.

BAJTÍN, Mikhail. Problemas de la poética de Dostoievski. México: FCE, 1986.

CHIERCHIA, Gennaro. The variability of impersonal subjects. En BACH, Emmon et al. (eds.). Quantification in Natural Languages. Netherlands: Kluwer Academic Publishers, 1995. p. 107-143.

CINQUE, Guglielmo. On si constructions and the theory of arb. Linguistic Inquiry, v. 19, n. 4, p. 521-581, 1988.

DUCROT, Oswald. El decir y lo dicho. Buenos Aires: Hachette, 1986.

FERNÁNDEZ RAMÍREZ, Salvador. Gramática española 4. El verbo y la oración. Madrid: Arco Libros, 1987.

GARCÍA NEGRONI, María Marta. La construcción media con se. Filología, XXIX, 1-2, p. 55-81, 1996.

GARCÍA NEGRONI, María Marta. Reformulación parafrástica y no parafrástica y ethos discursivo en la escritura académica en español. Contrastes entre escritura experta y escritura universitaria avanzada. Letras de Hoje, v. 44, p. 46-56, 2009.

GARCÍA NEGRONI, María Marta. Polifonía y subjetividad en el discurso académico en español. Estudos de Linguagens 2, p. 66-85, 2006.

HERNANZ, María Lluisa. En torno a los sujetos arbitrarios: la $2^{\underline{a}}$ persona del singular. En DEMONTE, Violeta et al. Estudios de lingüística de España y México. México: UNAM, Colegio de México, 1990. p. 151-178.
KERBRAT-ORECCHIONI, Catherine. O êthos em todos os seus estados. Análises do discurso hoje, v. 3, p. 117-135, 2010.

MAINGUENEAU, Dominique. El ethos y la voz de lo escrito. México: UAM, 1996. (Disponible <http://148.206.107.10/ biblioteca_digital/estadistica.php?id_host $=6 \&$ tipo $=$ ARTICUL O\&id=2004\&archivo=7-135-2004rqb.pdf\& titulo=El ethos y la voz de lo escrito>, visitado el 30 de julio de 2015).

MAINGUENEAU, Dominique. Problèmes d'ethos. Pratiques, n. 113/114, p. 55-67, 2002.

MENDIKOETXEA, Amaya. Construcciones con se: medias, pasivas e impersonales. En BOSQUE, Ignacio; DEMONTE, Violeta. Gramática Descriptiva de la lengua española. Madrid: Espasa Calpe, 1999. Cap. 26.

MENDIKOETXEA, Amaya. La semántica de la impersonalidad. En SÁNCHEZ LÓPEZ, Cristina. Las construcciones con se. Madrid: Visor, 2002. p. 235-271.

MIGUEL, Elena de. El aspecto en la sintaxis del español. Madrid: UAM, 1992.

MIGUEL, Elena de. El aspecto léxico. En BOSQUE, Ignacio; DEMONTE, Violeta. Gramática Descriptiva de la lengua española. Madrid: Espasa Calpe, 1999. Cap. 46.

MONTES GIRALDO, José Joaquín. El se del español y sus problemas. Estud. filol., n. 38, p. 121-137, 2003.

RAE. Nueva Gramática de la Lengua Española. Madrid: Espasa, 2009.

RAMÍREZ GELBES, Silvia. "Los títulos en los artículos científicos: por dónde andan los agentes". En el 1 O Congreso de Lecturas Múltiples, Universidad Nacional de Entre Ríos. Paraná, Entre Ríos, 2005a.

RAMÍREZ GELBES, Silvia. "Los títulos en los artículos científicos: por dónde andan los agentes". Ponencia presentada en el Primer Congreso de Lecturas Múltiples, Universidad Nacional de Entre Ríos. Paraná, Entre Ríos, 2005 b.

RAMÍREZ GELBES, Silvia. "Discurso académico, argumentación y ethos". Ponencia presentada en las Terceras Jornadas Internacionales de Educación Lingüística. Universidad Nacional de Entre Ríos. Concordia, Entre Ríos, 2006a.

RAMÍREZ GELBES, Silvia. "La lectura del agente: pasivas con se e impersonales con $s e$ ". En FLAWIÁ DE FERNÁNDEZ, N.; ISRAILEV, S. (comp.). Hispanismo: discursos culturales, identidad y memoria. Tucumán, FFyL-UNT, 2006b. Vol. III, p. 479-487.

RAMÍREZ GELBES, Silvia (2013). Cómo redactar un paper. La escritura de artículos científicos. Buenos Aires: Editorial Noveduc.

SÁNCHEZ LÓPEZ, Cristina (ed.). Las construcciones con se. Madrid: Visor, 2002b.

SÁNCHEZ LÓPEZ, Cristina. Las construcciones con se. Estado de la cuestión. En SÁNCHEZ LÓPEZ, Cristina. Las construcciones con se. Madrid: Visor, 2002a. p. 13-163.

SEGUNDO COLOQUIO LATINOAMERICANO DE ANALISTAS DEL DISCURSO. Resúmenes de comunicaciones. La Plata: UNLP, 1998.

SUÑER, Margarita. Non paradigmatic se's in Spanish. Tese doutoral, Indiana University, Bloomington, 1973 (citado por Sanchez López, 2002a). 


\section{Apéndice}

Encuesta presentada en Ramirez Gelbes (2006b).

(Se señala con cursiva negrita la opción seleccionada por

mayor cantidad de informantes en cada caso).

\section{REVISIÓN DEL CONCEPTO DE "EVALUACIÓN" NARRATIVA: UNA PERSPECTIVA GLOBAL}

La evaluación parece ser la sección de la narración de la cual depende el interés de una historia. En el trabajo de Labov (1972) sobre la narración oral, se establece un continuo con diferentes tipos de evaluación que van desde las más específicas hasta las más elaboradas, que el autor denomina evaluación externa, subordinación de la evaluación, acción evaluativa y suspensión de la acción.

En esta oportunidad, se propone un ordenamiento distinto de los diferentes tipos de evaluación. Se deja de lado su grado de elaboración para pasar a considerar la relación de las diferentes categorías evaluativas, tanto con las funciones del lenguaje como con las teorías sobre el punto de vista. De esta manera se logra una visión más coherente de esta sección de la narración.

1.a. ... se establece un continuo con diferentes tipos de evaluación...

a. Todos establecemos un continuo referido a la evaluación narrativa con diferentes tipos de evaluación.

b. Todos establecen un continuo referido a la evaluación narrativa con diferentes tipos de evaluación.

c. El autor de este texto establece un continuo referido a la evaluación narrativa con diferentes tipos de evaluación.

d. Alguien distinto del autor de este texto establece un continuo referido a la evaluación narrativa con diferentes tipos de evaluación. (se no locutivo)

1.b. ... se propone un ordenamiento distinto...

a. Todos proponemos un ordenamiento distinto de los diferentes tipos de evaluación.

b. Todos los que estudian la evaluación narrativa proponen un ordenamiento distinto de los diferentes tipos de evaluación.

c. El autor de este texto propone un ordenamiento distinto de los diferentes tipos de evaluación. (se locutivo)

d. Alguien distinto del autor de este texto propone un ordenamiento distinto de los diferentes tipos de evaluación. 1.c. Se deja de lado su grado de elaboración...

a. Todos dejamos de lado el grado de elaboración de los distintos tipos de evaluación narrativa para pasar a considerar la relación de las diferentes categorías evaluativas.

b. Todos dejan de lado el grado de elaboración de los distintos tipos de evaluación narrativa para pasar a considerar la relación de las diferentes categorías evaluativas.

c. El autor de este texto deja de lado el grado de elaboración de los distintos tipos de evaluación narrativa para pasar a considerar la relación de las diferentes categorías evaluativas. (se locutivo)

d. Alguien distinto del autor de este texto deja de lado el grado de elaboración de los distintos tipos de evaluación narrativa para pasar a considerar la relación de las diferentes categorías evaluativas.

1.d. ...se logra una visión más coherente...

a. De esta manera, todos logramos una visión más coherente de esta sección de la narración. (se inclusivo)

b. De esta manera, todos logran una visión más coherente de esta sección de la narración.

c. De esta manera, el autor de este texto logra una visión más coherente de esta sección de la narración.

d. De esta manera, alguien distinto del autor de este texto logra una visión más coherente de esta sección de la narración.

\section{EL CONCEPTO DE TEXTO EN LA TEORÍA DE LA MÚSICA}

Este trabajo se propone alcanzar una definición del concepto de "texto" dentro del campo especulativo de la Teoría Musical. Para lograrlo, se considerará la organización y estructura del texto musical desde un enfoque interdisciplinario, incorporando la perspectiva de la Lingüística textual. Se analizarán posibles paralelismos entre el texto del lenguaje natural y el texto del lenguaje musical, a fin de verificar si las categorías y los procedimientos de análisis que definen al primero pueden ser transferidos al segundo. 
El lenguaje musical no se analizará como un conjunto de estructuras abstractas, invariantes, potenciales, sino que se trabajará como un objeto complejo, dinámico, abierto, como texto, es decir, como producto de capacidades lingüísticas y cognitivas que permiten actualizar este lenguaje en contexto. Se podrá, entonces, abrir el área del lenguaje y la Teoría de la Música hacia el campo de los estudios del Discurso.

2.a. ...se considerará la organización y estructura del texto musical...

a. Para alcanzar una definición del concepto de texto en la Teoría Musical, todos consideraremos la organización y estructura del texto musical desde un enfoque interdisciplinario, incorporando la perspectiva de la lingüística textual.

b. Para alcanzar una definición del concepto de texto en la Teoría Musical, todos considerarán la organización y estructura del texto musical desde un enfoque interdisciplinario, incorporando la perspectiva de la lingüística textual.

c. Para alcanzar una definición del concepto de texto en la Teoría Musical, el autor de este texto considerará la organización y estructura del texto musical desde un enfoque interdisciplinario, incorporando la perspectiva de la lingüística textual. (se locutivo)

d. Para alcanzar una definición del concepto de texto en la Teoría Musical, alguien distinto del autor de este texto considerará la organización y estructura del texto musical desde un enfoque interdisciplinario, incorporando la perspectiva de la lingüística textual.

\section{2.b. Se analizarán posibles paralelismos...}

a. Todos analizaremos posibles paralelismos entre el texto del lenguaje natural y el texto del lenguaje musical. b. Todos analizarán posibles paralelismos entre el texto del lenguaje natural y el texto del lenguaje musical.

c. El autor de este texto analizará posibles paralelismos entre el texto del lenguaje natural y el texto del lenguaje musical. (se locutivo)

d. Alguien distinto del autor de este texto analizará posibles paralelismos entre el texto del lenguaje natural y el texto del lenguaje musical.

2.c. ... se trabajará como un objeto complejo...

a. Todos trabajaremos el lenguaje musical como un objeto complejo, dinámico, abierto, como texto.

b. Todos trabajarán el lenguaje musical como un objeto complejo, dinámico, abierto, como texto.

c. El autor de este texto trabajará el lenguaje musical como un objeto complejo, dinámico, abierto, como texto. (se locutivo)

d. Alguien distinto del autor de este texto trabajará el lenguaje musical como un objeto complejo, dinámico, abierto, como texto.

2.d. Se podrá, entonces, abrir el área del lenguaje y la Teoría de la Música...

a. Todos podremos, entonces, abrir el área del lenguaje y la Teoría de la Música hacia el campo de los estudios del Discurso. (se inclusivo)

b. Todos podrán, entonces, abrir el área del lenguaje y la Teoría de la Música hacia el campo de los estudios del Discurso.

c. El autor de este texto podrá, entonces, abrir el área del lenguaje y la Teoría de la Música hacia el campo de los estudios del Discurso.

d. Alguien distinto del autor de este texto podrá, entonces, abrir el área del lenguaje y la Teoría de la Música hacia el campo de los estudios del Discurso.

Recebido: 20 de agosto de 2015. 